for $x=-e$; $e$ being any number between $-\frac{a}{2}$ and $+\frac{a}{2}$. This cannot hold for $\sin \left(x \sqrt{ }, \frac{W}{C}\right)$, and therefore we must consider $E=0$. The solution therefore is restricted to $F \cdot \cos \left(x \sqrt{ } \frac{W}{C}\right)$. At the centre of the bar, where $x=\mathrm{o}$, this must $=B$. Therefore the solution is $y=B \cdot \cos \left(x \sqrt{\frac{W}{C}}\right)$.

Now here we have a very remarkable circumstance. It will be remembered that in the first investigation we arrived at a relation between $w$, the weight, and $b$, the greatest ordinate of the curve. But bere we find no relation whatever; and we come to this conclusion, that for the state of equilibrium fundamentally assumed, the degree of bulge of the bar is immaterial. And this agrees with plain reasoning : by varying the bulge of the bar, we vary in equal proportions, (I) the elasticity which depends on that bulge and on the general curvature, and (2) the distance of the line of action of $W$ from each point $p$, and its consequent angular momentum; and therefore, if they are equal for one degree of bulge, they

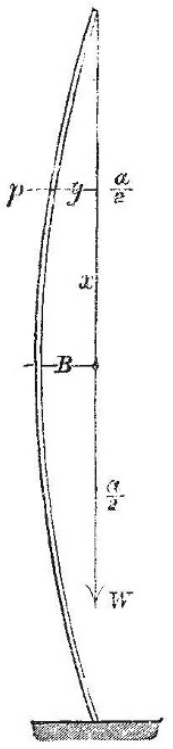

will be equal for every degree of bulge. The value of $B$, therefore is absolutely indeterminate.

But we do obtain one most important conclusion. When $x= \pm \frac{a}{2}, y$ must $=0$. And since, in the product $B \cdot \cos \left(\frac{a}{2} \sqrt{ } \frac{W}{C}\right)$, we are not permitted to make $B$ necessarily $=0$, we must make $\cos \left(\frac{a}{2} \sqrt{ } \frac{W}{C}\right)=0$. The simplest form of effecting this is by making $\frac{a}{2} \sqrt{ }_{C}^{W}=\frac{\pi}{2}$, or $W=C \cdot \frac{\pi^{2}}{a^{2}}$. Substituting for $C$ the value $\frac{a^{3} \cdot w}{48 \cdot b}$, which was found from the first investigation.

$$
W=\frac{\pi^{2}}{48} \cdot \frac{a}{b} \cdot w=0.205 \cdot \frac{a}{b} \cdot w
$$

and this defines the limiting value of the weight under which the curved bar can rest. If the weight be diminished, the curved bar will expand and lift it; if the weight be increased, that increased weight will crush down the curve.

It is important to observe that the furst and second in- vestigations apply to the same bar. And thus, in order to ascertain the limiting buckling force, we need only to ascertain by experiment on the same bar the amount of bend produced by any convenient transversal force.

In some cases, instead of making the first measure by application of the weight $w$ to act horizontally on the middle of the bar, it may be more convenient to make a measure of the vertical flexure of the bar (supported at its two end s in a free horizontal position), produced by its own weight. The following will be the corresponding theory.

(Third). Use the diagram of the first investigation, but substitute $c$ for $b$, and put $Z$ for the whole weight of the bar: and estimate the angular momentum round the point $p$. The reaction upwards of the force $\frac{Z}{2}$ at the pin produces $\frac{Z}{2} \times x$. The action downwards of the weight of bar included between the pin and the point $p$, which is $\frac{Z x}{a}$, will produce $\frac{Z x}{a} \times \frac{x}{2}$ or $\frac{Z x^{2}}{2 a}$. The combination of these produces the angular momentum $Z\left(\frac{x}{2}-\frac{x^{2}}{2 a}\right)$ upwards. The elastic force produces $C \times \frac{d^{2} y}{d x^{2}}$ downwards, where $C$ has the same value as in the first and second investigations. Making these equal, $C \cdot \frac{d^{2} y}{d x^{2}}=\frac{Z}{2 a}\left(a x-x^{2}\right)$. The first integration gives $C \cdot \frac{d y}{d x}=\frac{Z}{a}\left(\frac{a x^{2}}{4}-\frac{x^{3}}{6}\right)+$ ronstant. At the middle of the bar, where $x=\frac{a}{2}, \frac{d y}{d x}$ must $=0$; the constant therefore equals $-\frac{Z}{a}\left(\frac{a^{3}}{16}-\frac{a^{3}}{48}\right)$; and $C \cdot \frac{d y}{d x}=$ $\frac{Z}{a}\left(\frac{a x^{2}}{4}-\frac{x^{3}}{6}-\frac{a^{3}}{24}\right)$.

Integrating again, $C y=\frac{Z}{a}\left(\frac{a x^{3}}{12}-\frac{x^{4}}{24}-\frac{a^{3} x}{24}\right)+$ new constant. This is to be $\circ$ when $x=\frac{a}{2}$; the constant is found to be $+\frac{Z}{a} \cdot a^{4} \cdot \frac{5}{384}$. For the value when $x=0$, and consequently $y=c$, we have $C \cdot c=\frac{5 a^{3} \cdot Z}{384}$, or $C=$ $\frac{5 a^{3} \cdot Z}{384 \cdot c}$ Inserting this value of $C$ in the expression found in the second investigation,

$$
W=\frac{\pi^{2}}{a^{2}} \cdot \frac{5 a^{3} \cdot Z}{384 \cdot c}=\frac{5 \pi^{2}}{384} \cdot \frac{a}{c} \cdot Z=0.128 \frac{a}{c} Z,
$$

where (as before) $W$ is the limit of weight acting endwise on the bar, which the bar can bear without buckling.

If we wish roughly to introduce the consideration of the bar's weight, it will be sufficient to remark that at the lower part of the bar the whole weight of the bar is acting in conjunction with the weight $W ;$ and therefore, when we have computed the force (as above) we ought to deduct from that result the weight of the bar, and the residual will be the force which is permissible for action on the top of the bar.

G. B. A.

\section{THE LATE DR. VAN MONCKHOVEN}

I D Dr. Désiré Charles Van Monckhoven the scientific world has lost an able coadjutor, and his death is to be the more regretted in that he was taken from his many friends when almost in the prime of life. Van Monckhoven was born on September 25, 1834, and on September 25 of this year he died, having thus only traversed forty-eight years of the threescore-and-ten years 
to which there seemed every human probability he might reach. At an early age he turned his attention to scientific pursuits, and commenced his career as a chemist, the training for which so eminently fitte 3 him for the active part he took for the last twenty-five years in matters relating to photography. When scarcely o: age he wrote his "Traité Générale de Photographie," a new edition of which was called for almost year by year, its popularity being nearly unprecedented. Usually sound in his ideas, we may take it that much of the teaching of photochemistry has been propagated through the instrumentality of that work. Not only was Van Monckhoven an ardent experimentalist in the domain of chemical physics, but he also entered into all optical questions bearing on photography with a zeal which those alone who had the pleasure of his acquaintance can estimate. The Monckhoven solar enlarging apparatus is a standing record of his great ability in this department of science. At a very early period of his career he applied the spectroscope to record the effects of light on different inorganic and organic bodies, and his photographic researches on the spectra of gases occupied no inconsiderable portion of his time; his very latest published work, presented recently to the Académie des Sciences, being on the effect of temperature and pressure on hydrogen. Whilst Science, for herself, had charms for Van Monckhoven, yet he was able to put to commercial use much of the knowledge which he had acquired. For instance, he entered with enthusiasm into the mysteries of carbon printing, and established a factory for the production of the necessary tissue. Indeed the Monckhoven's tissue is the only one which enters into any sort of competition with that manufactured by the Autotype Company. Again to perfect the preparation of the latest photographic novelty-gelatine plates-he rushed into researches with all the ardour of an experimentalist, and having more or less mastered its intricacy, he established a manufactory for their commercial issue, and probably the Monckhoven plates are better known on the Continent than any other. Van Monckhoven, besides being the author of the treatise on photography, contributed many memoirs to various periodicals, amongst which we may name $L a$ Lumière, Le Bulletin Belge, and La Revue des deux Mondes. His style was vigorous, and everything he had to say was written with a terseness which many a busy scientific man may envy. In reviewing Van Monckhoven's life we cannot point to any great discovery or to any startling inventions he made, but he was one of those men who are so useful to science, giving, as it were, the decorations to the more solid building. We are sure that thougb there may be greater names, there is scarcely one which is more universally known than his, and whose loss will be more universally felt. There are not many who can claim to be dictinguished as an astronomer, a chemist, an optician, and photo-chemist, Van Monckhoven could make good his claim to such distinction, and withal to be a busy man in the world of commerce.

Within a short time of his death he was engaged in an important research on the influence of pressure and temperature on the spectra of gases, in which he had introduced quite a new method of attack, and one which promised to be of great value.

\section{THE CHEMISTRY OF THE PLANTE AND FAURE ACCUMULATORS}

\section{PART IV.-The Function of Sulphate of Lead}

I $N$ our previous communications on the chemistry of the lead and peroxide batteries we have frequently remarked on the formation of lead sulphate and its importance in the history of a cell.

in Part I. (Nature, vol. xxy. p. 221) we showed that the local action that takes place at first energetically between the metallic lead and the peroxide is gradually diminished by the formation of sulphate of lead.

In Part II. (vol. xxv, p. 46I) we stated that in the original formation of a Faure cell sulphate of lead is oxidated on the one plate and reduced on the other. We also described an experiment in which two platinum plates were covered with lead-sulphate, immersed in dilute sulphuric acid, and placed in the circuit of a galvanic current; the result being that "the white sulphate was decomposed to a large extent on each plate, the positive being covered with deep chocolate-coloured peroxide, the negative with grey spongy lead."

In Part III. (vol. xxvi. p 25I) we showed that on the discharge of a cell, lead sulphate is the ultimate product on both plates.

It might naturally be inferred from our previous statements that in the re-charging of a cell this lead sulphate would be oxidated on the one plate and reduced on the other as in the original formation. This matter, however, has given rise to so.ne controversy. All subsequent experimenters admit the oxidatzon of the lead-sulphate, but Dr. Oliver Lodge could not obtain any reduction of it, when pure sulphate was employed. Sir William Thomson also, when experimenting, with two platinum plates and layers of sulphate, obtained only a doubtful indication of reduced metal. The question as to whethes the sulphate is reduced or not on re-charging a Faure cell is one of vital importance; for if the sulphate formed at each discharge accumulates on the positive plate it would clog up the space, and, what is perhaps worse, a fresh surface of the lead would have to be oxidated (or rather, converted into sulphate) at each dis sharge. Thus the positive plate will be continually corroded, and its life will be limited.

We have already replied to Dr. Lodge in NATURE (vol. xxvi. p. 342), but we thought it desirable to repeat the experiment with the platinum plates, especially with a view to determine whether the reduction was effected slowly or with any rapidity. We fastened 20 grms. of the white sulphate upon a negative plate by binding it round tightly with parchment-paper, placed it vertically in the sulphuric acid, and passed a continuous current of somewhat under an Ampère. The hydrogen was at no time wholly absorbed-indeed the greater part of it certainly escaped--but after the lapse of twenty-four hours, small patches of grey metallic lead became distinctly visible through the wet parchment-paper; and these gradually spread in an irregulur manner. At the end of ten days it was found that the whole of the sulphate, except a few small patches on the surface, was reduced to a grey spongy mass. Although there could be no reasonable doubt that this was metallic lead, a portion of it was tested caemically, and proved to be such.

It thus appears that the reduction of the pure sulphate: of lead is an absolute fact, although it does not take place so easily as the oxidation.

In an actual cell the sulphate of lead is of course mixed with other bodies. Thus, in the formation of a Faure battery, the minium is converted by the sulphuric acid more or less completely into peroxide of lead and sulphate. We have already described an experiment in which 4489 c.c. of hydrogen were absorbed on a plate, the materials of which were capable of absorbing only 4574 c.c., if the whole sulphate as well as the peroxide was reduced In our note-book we have the particulars of four other experiments made in each case with the same, or nearly the same, amount of material, in which 4199, 4575,4216 , and 4387 c.c. respectively were absorbed, although perhaps in not one of these cases was the experiment continued until the action was absolutely complete. As, however, it may be objected that the amount of sulphate produced upon these plates was an unknown quantity, we have in a recent experiment treated the 